to all those tested. All HCV RNA positive were offered a clinic review 10 days after testing to commence treatment with a pangenotypic antiviral.

Results Of the 307 residents in the prison at the time of the event, 305 (99\%) accepted BBV testing. A total of 98 (32\%) were HCV antibody positive, of these 23 were HCV RNA detected (23\% of HCV Ab pos and $8 \%$ of all tested) in keeping with active HCV. One resident was HIV positive (known) and 4 had positive syphilis serology. None were HBsAg positive. Of the $23 \mathrm{HCV}$ RNA positive residents, 3 were already on antiviral treatment, 17 commenced antivirals and 3 were released before treatment could be initiated (contact planned in the community). One patient was suspected of having cirrhosis. Of the $75 \mathrm{HCV}$ antibody positive but RNA negative residents 40 (53\%) were known to have received antiviral treatment already and achieved sustained virological response and $10(13 \%)$ were currently on treatment. Feedback from residents and staff on the way the HITT was conducted was good. A point of care HCV RNA testing machine is now being used to identify $\mathrm{HCV}$ infection among new residents to try and maintain 'elimination'.

Conclusions A high intensity test and treat weekend coupled with quick access to antiviral treatment for HCV is a highly effective way to 'eliminate' HCV within a prison. However, these sessions require meticulous planning in order to be successful.

Acknowledgment All the staff and residents of HMP Low Newton and the Hepatitis C Trust.

\section{O66 OUTCOME OF INVESTIGATION FOR SUSPECTED MALIGNANCY IN PATIENTS WITH IRON DEFICIENCY ANAEMIA WITHOUT GASTROINTESTINAL SYMPTOMS}

${ }^{1}$ Gwilym Webb*, 'Thomas Chapman, ${ }^{2}$ Brian Shine, ${ }^{1}$ Jack Satsangi, ${ }^{1}$ Antony Ellis. ${ }^{1}$ Translational Gastroenterology Unit, NIHR Oxford Biomedical Research Centre, Oxford, UK; ${ }^{2}$ Nuffield Department of Clinical Sciences, University of Oxford, Oxford, UK

\subsection{6/gutjnl-2020-bsgcampus.66}

Introduction Iron deficiency anaemia (IDA) without additional gastrointestinal (GI) symptoms is common. Due to the association with GI malignancy, investigation of the upper and lower GI tract is recommended in older adults with IDA. However, rates of specific diagnoses are incompletely defined, and criteria to permit rationalisation of investigation are unclear. The yields of repeated investigation and of testing for coeliac disease (CD) are also uncertain.

Methods Cohort study derived from a prospectively collected referrals database of patients with IDA and suspected cancer from two UK hospitals in a single NHS Trust over a 52month period.

Results 5702 consecutive referrals were assessed and, after exclusions, 2035 patient referrals for IDA without additional GI symptoms were assessed. 1118 (54.9\%) were women; median age was 74 years (IQR 66-81).

Cancer was diagnosed in 147 (7.2\%) and luminal GI cancer in $120(5.9 \%)$. For luminal cancers, the site was colorectal in $103(5.0 \%$ of all patients), gastric in $11(0.5 \%)$ and oesophageal in $6(0.3 \%)$. Other diagnoses made in $\geq 1 \%$ were benign upper GI ulceration in 77 (3.8\%), ulcerative colitis in $35(1.7 \%)$, and $\mathrm{CD}$ in $21(1.0 \%)$. No major diagnosis was found in 1706 (83.8\%).
Those with luminal cancer were older (78 vs 74 years; $\mathrm{p}<0.001$ ), more anaemic (Hb 89 vs $101 \mathrm{~g} / \mathrm{L} ; \mathrm{p}<0.001)$, had higher CRP (38 vs $5.8 \mathrm{mg} / \mathrm{L}$; p<0.001), lower ferritin (14 vs $15 \mu \mathrm{g} / \mathrm{L} ; \mathrm{p}=0.012$ ), lower transferrin saturation (7 vs $9 \%$; $\mathrm{p}<0.001)$, lower MCV (79.8 vs $83.2 \mathrm{fL}$; $\mathrm{p}<0.001)$ and lower MCHC (296 vs $302 \mathrm{~g} / \mathrm{L} ; \mathrm{p}<0.001$ ), and were more likely to be male $(53.7 \%$ vs $44.5 \%$; $=0.048)$. The single most discriminatory variable for predicting luminal cancer was haemoglobin deficit (AUROC 0.64).

After multivariable analysis, age (RR 1.56/10 years, 95\%CI 1.23-1.99, $\mathrm{p}<0.001$ ); elevated CRP (RR 1.10/10 mg/L, 1.071.14, p<0.001); lower MCV (RR 0.47/10 fL, 0.31-0.71, $\mathrm{p}<0.001$; and male sex (RR 1.92, 1.16-3.18, $\mathrm{p}=0.011)$ were significant for risk of malignancy.

Of 142 patients (7.0\%) referred more than once within the study period, just two had luminal gastrointestinal cancer $(1.4 \% ; \mathrm{p}=0.027$ vs first referrals).

Only $21(1.4 \%)$ of 1485 patients who underwent duodenal biopsy had histology compatible with $\mathrm{CD}$; for serology, 7 of 557 patients $(1.3 \%)$ were positive.

Conclusions This study, to our knowledge the largest such cohort yet reported, provides data that will aid both patient counselling and the development of referral and investigation pathways for IDA. Repeated investigation of IDA and duodenal biopsy for CD in IDA without GI symptoms have limited yields.

\section{THE DEVELOPMENT OF A WEB-BASED APPLICATION TO PREDICT THE RISK OF GI CANCER IN IDA}

1,2Orouba Almilaij*, ${ }^{3}$ Vegard Engen, ${ }^{1}$ Peter Thomas, ${ }^{2}$ Jonathon Snook, ${ }^{1}$ Clinical Research Unit, Bournemouth University, Bournemouth, UK; ${ }^{2}$ Gastroenterology Unit, Poole Hospital NHS Foundation Trust, Poole, UK; ${ }^{3}$ Department of Computing and Informatics, Bournemouth University, Bournemouth, UK

\subsection{6/gutjnl-2020-bsgcampus.67}

Introduction Gastrointestinal (GI) malignancy is a common finding in iron deficiency anaemia (IDA), with a prevalence of about $8 \%$. We have previously reported and validated an algorithm for predicting the risk of GI malignancy in IDA - the IDIOM score. This was derived by logistic regression analysis based on four independent and objective clinical parameters age, sex, mean corpuscular volume (MCV), and haemoglobin concentration $(\mathrm{Hb})$. To facilitate the clinical use of this algorithm, a software application has been developed, with a view to providing free and simple access to healthcare professionals in the UK.

Methods A detailed requirements analysis for intended users of the application revealed the need for an automated tool in which anonymised, individual, patient data is entered and GI cancer risk is calculated and displayed. The solution needed to be user-friendly and platform independent, and needed to facilitate future communication with the development team. Human-centred design (HCD) was employed to develop the solution, focusing on the users and their needs, whilst ensuring that they are provided with sufficient details to appropriately interpret the risk score. To evaluate usability, standard usability questionnaire applied. Participants include healthcare professionals such as IDA nurse specialists, gastroenterologists, etc.

Results Predict GI Cancer in IDA has been developed using $\mathrm{R}$ Shiny as a web-based application enabling access from 